\title{
ANÁLISE BIBLIOMÉTRICA DE ALTERNATIVAS PARA REMOÇÃO DE NUTRIENTES DE ÁGUAS RESIDUAIS NAS BASES SCOPUS, WEB OF SCIENCE E SCIELO
}

\author{
BIBLIOMETRIC ANALYSIS OF ALTERNATIVES FOR REMOVING NUTRIENTS FROM \\ WASTEWATER IN SCOPUS, WEB OF SCIENCE AND SCIELO DATABASES
}

\section{Eliza Rocha Moreto ${ }^{1 *}$, Paulo Sérgio da Silva Porto ${ }^{2}$, \& Rodrigo Randow de Freitas ${ }^{3}$}

123 Universidade Federal do Espírito Santo, Rodovia BR 101 Norte, Km. 60, Bairro Litorâneo, CEP 29932-540, São Mateus. ${ }^{1 *}$ moretoeliza@gmail.com ${ }^{2}$ paulo.porto@ufes.br ${ }^{3}$ rodrigo.r.freitas@ufes.br

\section{ARTIGO INFO.}

Recebido em: 17.10.2020

Aprovado em: 18.11.2020

Disponibilizado em: 17.12 .2020

Palavras-chave:

Eutrofização; tratamentos; efluentes.

\section{KEYWORDS:}

Eutrophication; treatment; effluent.

*Autor Correspondente: Moreto, E. R.

\section{RESUMO}

O crescimento exponencial da população e o uso excessivo dos recursos hídricos vêm gerando problemas tanto ambientais, como a contaminação de rios, mares e solo, quanto para as pessoas no quesito escassez da água. Logo o presente trabalho se justifica pela importância de geração de informações sobre as ações que podem ser realizadas para reaproveitamento das aguas contaminadas. Assim, objetiva-se a realizar uma análise bibliométrica sobre quais são as principais alternativas de remoção de nutrientes em corpos d'água utilizando as bases Scopus, Web of Science e SciELO. Para isso, realizou-se uma revisão de literatura para fundamentação teórica e identificação das bases de dados, definição das palavras-chave que seriam utilizadas nas bases de dados e aplicação de filtros para selecionar os artigos que condiziam com o tema e consequentemente formar o portfólio de trabalhos mais relevantes. A partir disso, foram obtidos 9 artigos na base Scopus, 5 na WoS e 3 na SciELO para composição do portfólio. Com base na análise bibliométrica realizada nas bases Scopus, Web of Science e SciELO foi possível visualizar o crescimento de pesquisas visando à solução de problemas ambientais causados pela liberação de nutrientes no meio ambiente.

\begin{abstract}
The exponential growth of the population and the excessive use of water resources have been generating environmental problems, such as the contamination of rivers, seas and soil, as well as for people with regard to water scarcity. Therefore, the present work is justified by the importance of generating information about the actions that can be taken to reuse contaminated water. Thus, the objective is to carry out a bibliometric analysis on what are the main alternatives for removing nutrients from water bodies using the Scopus, Web of Science and SciELO bases. For this, a literature review was carried out for theoretical foundation and identification of the databases, definition of keywords that would be used in the databases and application of filters to select the articles that matched the theme and consequently form the portfolio relevant work. From this, 9 articles were obtained in the Scopus database, 5 in WoS and 3 in SciELO to compose the portfolio. Based on the bibliometric analysis carried out on the Scopus, Web of Science and SciELO databases, it was possible to visualize the growth of research aimed at solving environmental problems caused by the release of nutrients into the environment.
\end{abstract}


Citação (APA): Moreto, E. R., Porto, P. S. da S., \& Freitas, R. R. de. (2020). Análise bibliométrica de alternativas para remoção de nutrientes de águas residuais nas bases Scopus, Web of Science e Scielo. Brazilian Journal of Production Engineering, 6(7), 133-149.

\section{INTRODUÇÃO}

O crescimento exponencial da população e o uso excessivo dos recursos hídricos vêm gerando problemas tanto ambientais, quanto para as pessoas, devido ao grande aumento no consumo nos últimos 20 anos (São Paulo, 2020). Além do uso excessivo da água, a contaminação da mesma, que pode ser causada por lixões, atividades inadequadas de armazenamento, efluentes e resíduos em atividades industriais, como indústrias químicas, petroquímicas, metalúrgicas, dentre outros, são inequívocos e alarmantes (São Paulo, 2016). Destacam-se as elevadas quantidades de nutrientes de nitrogênio, amônia e fósforo (Pantano, et al., 2016). O acúmulo desses nutrientes gera o fenômeno chamado de eutrofização, que provoca o nascimento de algas e cianobactérias, dificultando a vida aquática e consequentemente provocando o desequilíbrio do sistema (Trindade, \& Mendonça, 2014).

Atualmente as empresas sentem a necessidade de se adequarem quanto ao tratamento das águas residuais geradas, devido à obrigatoriedade das leis e normas ambientais, que implicam em punições de corresponsabilidade ambiental (Resolução Conama No 430/2011). As leis que regem a legislação ambiental são: a Lei 12.305/2010 - Institui a Política Nacional de Resíduos Sólidos (PNRS) e altera a Lei 9.605/1998 - que estabelece diretrizes à gestão integrada e ao gerenciamento ambiental adequado dos resíduos sólidos e propõe regras para o cumprimento de seus objetivos em amplitude nacional interpretando a responsabilidade como compartilhada entre governo, empresas e sociedade. Na prática, definindo que todo resíduo deve ser processado apropriadamente antes da destinação final e que o infrator está sujeito a penas passivas, inclusive, de prisão. Destaca-se, por exemplo, a possibilidade de penalização das pessoas jurídicas no caso de ocorrência de crimes ambientais.

Além das leis tem-se a imagem que a empresa passa para seus consumidores; hoje em dia os clientes estão desejando comprar produtos confeccionados de forma sustentável e que não causam nenhum dano ao meio ambiente (Garcia, 2016). Um exemplo positivo disso é a empresa da Natura, que se mostrou preocupada com o meio ambiente mostrando isso em seus produtos (sacolas de papel e extração de matéria prima consciente) e tornando a sustentabilidade o direcionador de seus negócios (Natura, 2018).

Logo o presente trabalho se justifica pela importância de geração de informações sobre as ações que podem ser realizadas para reaproveitamento de efluentes e a facilidade que este trabalho pode proporcionar a indivíduos interessados, propiciando quais caminhos para se seguir. Assim, objetiva-se realizar uma análise bibliométrica sobre as principais alternativas de remoção de nutrientes em corpos d'água no quesito ambiental utilizando as bases Scopus, Web of Science e SciELO.

\section{Metodologia}

A pesquisa baseou-se em um tratamento quantitativo, sendo realizados estudos com características objetivas de quantificação, tais como, filtros estatísticos, contagem de citações e análise de gráficos. A mesma também é tratável como exploratório-descritiva, já que objetiva explicar e analisar o que está ocorrendo com os dados coletados (Souza, 2013).

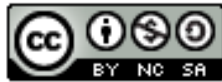



de águas residuais nas bases Scopus, Web of Science e Scielo. Brazilian Journal of Production Engineering, 6(7), 133-149.

Para uma melhor familiaridade com o tema, foi efetuado uma revisão de literatura para fundamentação teórica e uma identificação das bases de dados que seriam utilizadas e posteriormente. A partir disso foi definido as 3 palavras-chaves que mais abrangiam o tema nas bases escolhidas. A Scopus foi escolhida, pois ela faz uso de ferramentas bibliométricas para acompanhamento, análise e visualização de pesquisas, além de ser considerada a maior base de dados de resumos, contendo mais de 22.000 trabalhos e 5.000 editores por todo o mundo (Elsevier, 2020). Já base de dados Web Of Science foi selecionada devido à facilidade que ela proporciona na análise dos dados em pesquisas bibliométricas, a qualidade dos trabalhos ali contidos e por ser caracterizada como a mais antiga e de ampla cobertura de dados de citações e dados bibliográficos (Boyle, \& Sherman, 2006). Por último, optou-se pela base SciELO por ser uma base de dados nacional e por promover visibilidade e acessibilidade às publicações científicas da América Latina (Meneghini, 2003).

Logo que as palavras-chave foram definidas e aplicadas nas bases de dados, deu-se início a elaboração dos filtros em que os trabalhos selecionados iriam passar, para enfim estruturar o portfólio de publicações. Na primeira triagem foram selecionados os trabalhos apenas do tipo artigo, pois estes são os que possuem maior relevância cientifica, datados entre entre os anos de 2015 e 2020, devido serem mais atuais e possivelmente seguirem as mudanças tecnológicas. Após essa seleção, os artigos foram transferidos para uma planilha eletrônica, para uma melhor visualização e análise.

Num segundo momento, realizou-se a leitura dos resumos para determinar quais artigos condiziam com o tema dentre os artigos. Os trabalhos cujo conteúdo estava fora do desejado para a pesquisa, foram excluídos. Após leitura dos resumos, foi elaborada uma nuvem de palavras utilizando a ferramenta Word Cloud (Wordcounter, 2015) com as áreas de estudo em que os artigos foram publicados. Essa ferramenta converte certa quantidade de palavras escolhidas pelo autor em uma nuvem; o dimensionamento dessas palavras é proporcional à frequência com que elas aparecem, ou seja, quanto mais a palavra for citada, maior ela será. Segundo Cui (2010), as nuvens de palavras são utilizadas como ponto inicial para uma análise mais profunda.

Posteriormente, sucedeu-se a coleta dos fatores de impacto das revistas cientificas na plataforma JCR (Journal Citation Report) para a base Scopus, WoS e Sucupira Qualis para a base SciELO; nessa etapa objetivou-se excluir do portfólio as revistas que não possuíam qualificação.

Outro filtro de seleção utilizado foi o método de Lacerda (2012), que utiliza um valor de corte para os artigos mais citados, ou seja, com os artigos em ordem decrescente conforme a quantidade de citações é selecionada $85 \%$ do total de citações obtidos quando somadas.

Conseguinte, foi definido um número amostral válido para uma nova seleção de artigos. Segundo Cay e Uyan (2013), a dimensão de uma amostra (número de artigos necessários) provém do tamanho da população (total de artigos) e o grau de confiabilidade que se deseja. Para assegurar a validade da amostra e a representatividade dos dados coletados foi utilizado então a Equação 1 (Dupont, \& Plummer, 1990), para determinar o tamanho da amostra.

\section{@ $\odot \Theta \odot$}


Citação (APA): Moreto, E. R., Porto, P. S. da S., \& Freitas, R. R. de. (2020). Análise bibliométrica de alternativas para remoção de nutrientes de águas residuais nas bases Scopus, Web of Science e Scielo. Brazilian Journal of Production Engineering, 6(7), $133-149$.

Equação 1. Equação que estabelece o tamanho da amostra

$$
n=\frac{Z^{2} \cdot\left(\frac{x}{n}\right) \cdot\left[1-\left(\frac{x}{n}\right)\right] \cdot N}{(N-1) \cdot e^{2}+Z^{2} \cdot\left(\frac{x}{n}\right) \cdot\left[1-\left(\frac{x}{n}\right)\right]}
$$

Fonte: Dupont e Pulmmer, 1990.

No qual,

- n é a dimensão da amostra que se deseja;

- N é o tamanho da população;

- e é o erro amostral;

- x/n é a proporção estimada do item pesquisado na amostra (\%);

- Z é o valor da abscissa da curva normal associada ao nível de confiança (Barbetta, 2007).

Considerando um erro amostral de $10 \%$, um valor de $50 \%$ para $\mathrm{x} / \mathrm{n}$, pois não se tem uma proporção estimada e um nível de confiança de $90 \%$.

Posteriormente, com a seleção dos artigos completa, foram analisados às informações ali contidas sobre os avanços que o tema está sofrendo em meio à comunidade acadêmica através dos resultados e conclusões de cada artigo do portfólio. E por fim, elaborou-se uma matriz SWOT (Referência, Ano), para identificação dos pontos positivos e negativos sobre as projeções futuras para o desenvolvimento científico, ou seja, as ameaças, fraquezas, oportunidades e forças. Para uma melhor visualização dos filtros que foram aplicados ao longo do trabalho foi feito um fluxograma (Figura 1).

Figura 1. Fluxograma do processo de seleção.

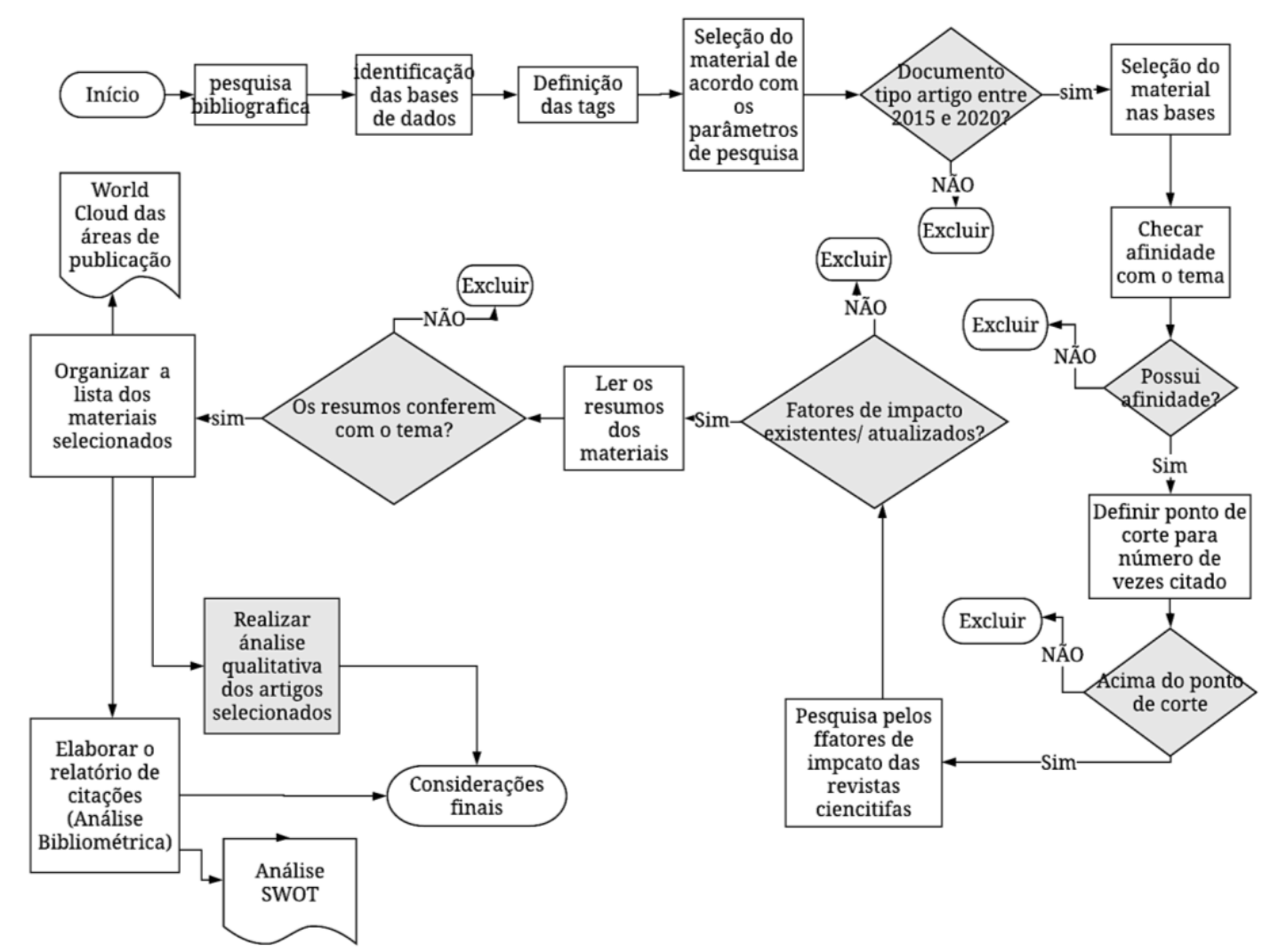

Fonte: Autores, 2020. 


\section{RESULTADOS}

A partir da definição dos termos de busca que mais abrangiam o tema, chegou-se as seguintes palavras-chave, "eutrophication", "treatment" e "efluente" com incialmente 1013 trabalhos na Scopus, 683 na Wos e 17 na SciELO. Posteriormente ao delimitar-se as bases para os anos de 2015 a 2020 e trabalhos do tipo artigo, obteve-se 302 artigos na Scopus, 275 na WoS e 6 na SciELO.

Com os artigos selecionados, pôde-se observar um crescimento do número de publicações ao longo dos anos selecionados na pesquisa, em todas as bases, como exposto no Gráfico 1.

Gráfico 1. Quantidade de artigos por ano

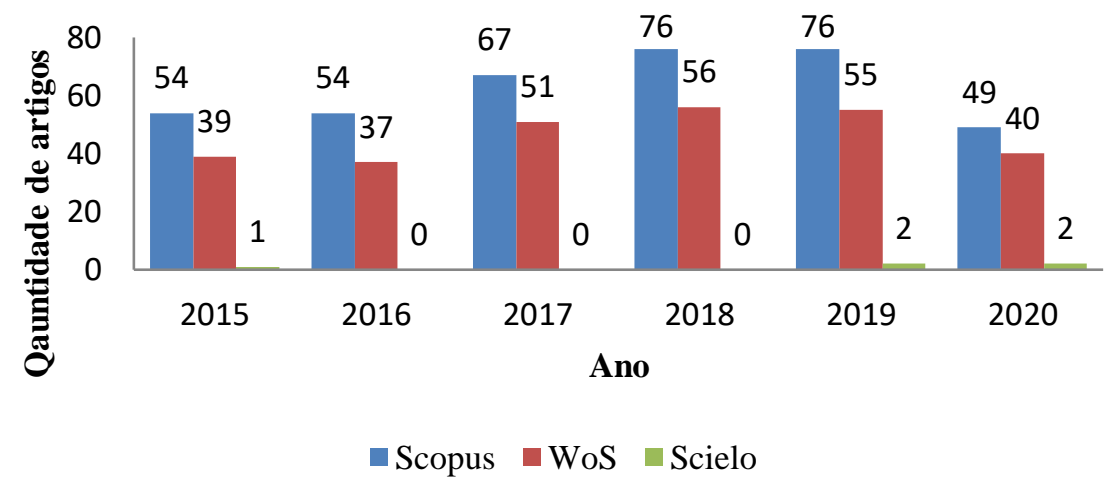

Fonte: Autores, 2020.

O mesmo aconteceu no estudo de Moreira, Filho e Mueller (2015), que ao realizarem uma análise das características da produção científica dos grupos de pesquisa do CNPq/DGP nas áreas de Ciência da Informação e Museologia entre os anos de 1992 e 2012 e constataram que as publicações científicas cresceram cerca de 940\%, passando de 110 trabalhos em 1992, para 1.140 em 2012.

Outro fator importante é a quantidade de artigos publicados por base de dados como vemos no gráfico 1; a scopus é a base com a maior quantidade de publicações (57\%), logo após vem a WoS com $42,2 \%$ e por ultimo a SciELO com $0,75 \%$. É perceptível uma diferença muito grande entre as bases internacionais e a nacional e segundo Guedón (2010), isso é possível pois pesquisadores acreditam que bases internacionais possuem uma maior visibilidade devido o inglês ser um idioma universal e ambas as bases, tanto Scopus quanto WoS serem bases conhecidas e acessadas no mundo todo.

Quanto a quantidade de publicações por países, tem-se que a maioria das publicações são realizadas nos mesmos países. Na Scopus, dos 61 países com publicações, China, EUA e Brasil representem 47,51\%; na WoS dos 25 países representados, China, EUA e Brasil dominam com $45 \%$ e na SciELO $100 \%$ das publicações advém do Brasil. 

de águas residuais nas bases Scopus, Web of Science e Scielo. Brazilian Journal of Production Engineering, 6(7), 133-149.

Gráfico 2. Quantidade de publicações por países e Bases estudadas.

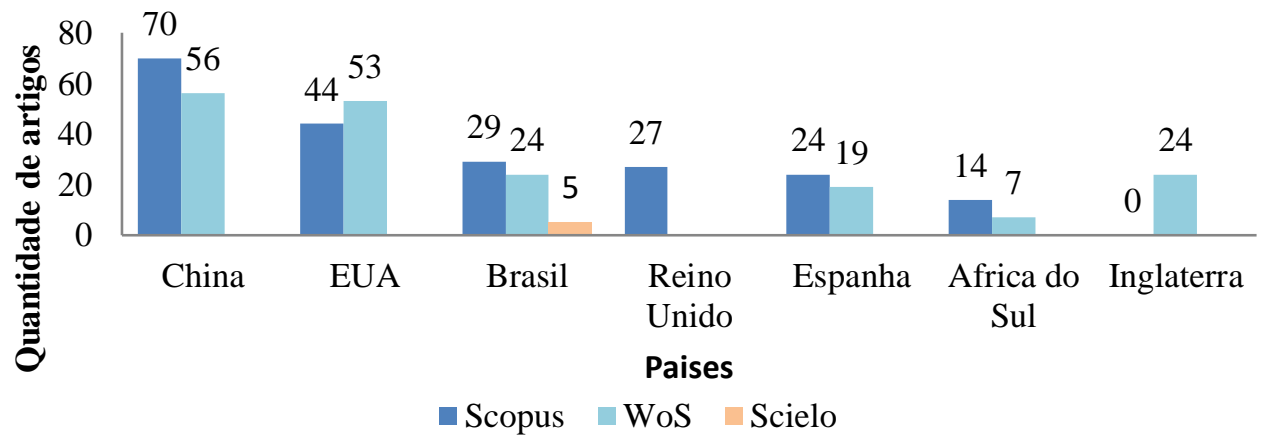

Fonte: Autores, 2020.

Outra questão analisada foi à quantidade de artigos por área de estudo; Essa análise auxilia na determinação do foco e direção em que a pesquisa está sendo conduzida. Logo, tem-se que na base Scopus, $45 \%$ das publicações de pesquisas são voltadas para ciências ambientais, 14\% para engenharias e 9,17\% para ciências agrarias e biológicas. Com relação a Web of Science, $29 \%$ dos artigos estão inclusos na área de ciências ambientais, 17\% em engenharia ambiental e 16,4\% em recursos hídricos. Por último, na base SciELO, 37,5\% dos artigos estão identificados como multidisciplinares, $37,5 \%$ são de conhecimentos voltados para ciências agrárias e 12,5\% para ciências biológicas.

A fim de se evidenciar as áreas de pesquisa (periódicos) em que os artigos foram publicados e reforçar o último filtro aplicado (quantidade de artigos por área de estudo) fez-se a utilização da ferramenta Word Cloud (Figura 2).

Figura 2. Áreas de conhecimento em que os artigos selecionados foram publicados

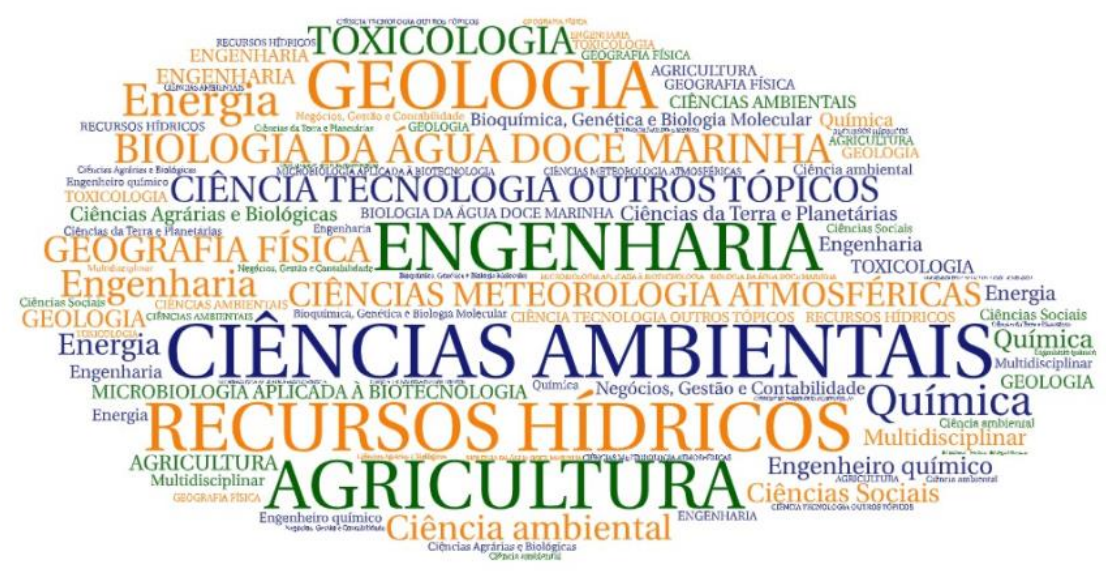

Fonte: Autores, 2020.

Outro aspecto analisado foi o local de publicação do estudo (periódicos). Como o tema desta pesquisa está relacionado a buscar alternativas de soluções para problemas ambientais globalmente, existem muitos periódicos com maior quantidade de publicações na temática. $\mathrm{Na}$ WoS o periódico "Science of the total environment", contem 14 artigos e na scopus o mesmo contem 22; Com relação a revista "Water research, a mesma possui 15 artigos na WoS e 18 artigos na scopus (Gráfico 3). Já na SciELO a "Revista Brasileira de Engenharia

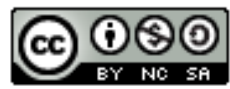


Citação (APA): Moreto, E. R., Porto, P. S. da S., \& Freitas, R. R. de. (2020). Análise bibliométrica de alternativas para remoção de nutrientes de águas residuais nas bases Scopus, Web of Science e Scielo. Brazilian Journal of Production Engineering, 6(7), 133-149.

Agrícola e Ambiental" possui 2 artigos, "Engenharia Sanitária e Ambiental”, "Brazilian Journal of Microbiology" e "Anais da Academia Brasileira de Ciências" com 1 artigo.

Gráfico 3. Quantidade de publicações por periódicos

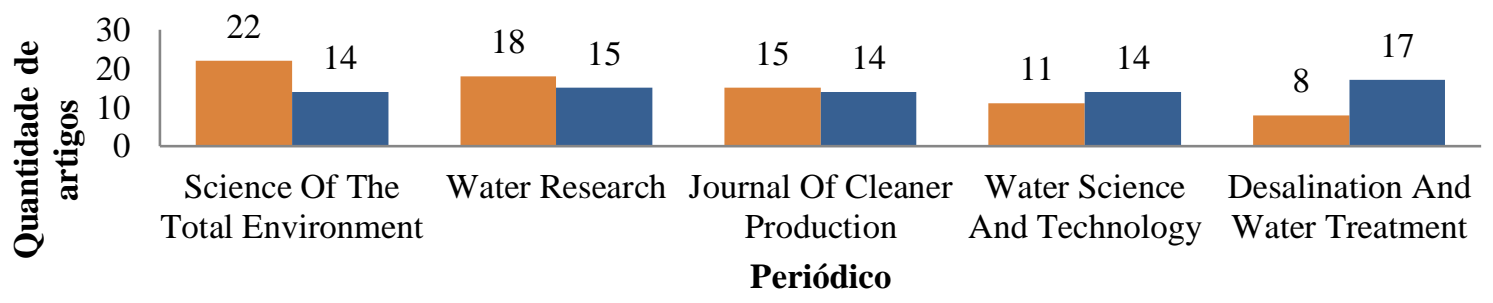

$\square$ Scopus $\square$ WoS

Fonte. Autores, 2020.

Ao analisar a relação entre periódicos, fator de impacto e artigos publicados, foi possível evidenciar que quanto maior a quantidade de publicações e citações, maior é o fator de impacto (FI). Segundo Strehl (2005), o FI proporciona uma maior visibilidade e relevância aos periódicos que o contém, já que ele garante notoriedade das publicações dentro e fora da comunidade acadêmica. A partir dessa constatação, o periódico que possui o melhor FI nas bases internacionais (WoS e scopus) foi o "Water Research" com 9,13; na sequência tem-se o periódico "Science of the total environment" com um FI de 7,86, "Journal of Cleaner production" com 7,25 .

Importante mencionar que o FI de um periódico é calculado utilizando a quantidade de citações dos 2 últimos anos, dividida pela quantidade de artigos que foram citados (Tabela 1). Logo ao se investigar o porquê do periódico "Water Research" ter o maior FI, com uma menor quantidade de publicações, têm-se que a revista "Science of the total environment", mesmo tendo uma maior quantidade de publicações e quase o dobro de citações (anos de 2017 e 2018), teve uma maior diversificação de artigos que foram citados.

Tabela 1. Cálculo de FI

\begin{tabular}{c|c|c|c}
\hline Periódicos & $\begin{array}{c}\text { Quantidade de citações } \\
\text { em 2017 e 2018 }\end{array}$ & $\begin{array}{c}\text { Quantidade de artigos } \\
\text { citados em 2017 e 2018 }\end{array}$ & $\begin{array}{c}\text { Fator de } \\
\text { impacto (FI) }\end{array}$ \\
\hline $\begin{array}{c}\text { Science of the total } \\
\text { environment }\end{array}$ & 23827 & 3030 & 7,86 \\
\hline Water Research & 15001 & 1643 & 9,13 \\
\hline
\end{tabular}

Fonte: Autores, 2020.

Para a base SciELO, a plataforma de verificação dos FI é a Sucupira, cujos resultados obtidos foram A2 para os periódicos Engenharia Sanitária e Ambiental e a Revista Brasileira de Engenharia Agrícola e Ambiental e B1 para as revistas Brazilian Journal of Microbiology e Anais da Academia Brasileira de Ciências. Percebe-se que a quantidade de publicações em periódicos brasileiros sobre este tema é muita pequena, apesar de possuir um fator de impacto bom, já que revistas renomadas consideram as classificações A1, A2, B1 e B2 satisfatórias; Uma explicação para esse fato é que os pesquisadores nacionais escolhem publicar seus trabalhos em revistas estrangeiras devido à importância do FI da JCR como critério de

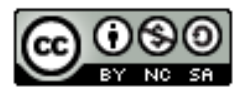


Citação (APA): Moreto, E. R., Porto, P. S. da S., \& Freitas, R. R. de. (2020). Análise bibliométrica de alternativas para remoção de nutrientes de águas residuais nas bases Scopus, Web of Science e Scielo. Brazilian Journal of Production Engineering, 6(7), 133-149.

avaliação da produção científica e a visibilidade que o mesmo da por ser da língua estrangeira (Mugnaini, 2006).

Continuando a aplicação de filtros de seleção, a próxima fase da análise foi à leitura dos resumos para identificação dos artigos que condiziam com o tema, onde se encontrou 47 artigos na Scopus, 36 na WoS e 3 na SciELO; o restante dos artigos foram excluídos por serem de temas não condizentes com o estudo.

Quando somada a quantidade citações dos artigos selecionados no ultimo filtro por base temse na Scopus 668 citações, 359 na WoS e 0 na SciELO; como a quantidade de artigos condizentes com o tema na SciELO foi muito pequena (3 artigos), os próximos filtros (método de Lacerda e estabelecimento do tamanho amostral) não foram utilizados nessa base. Aplicando o método de corte de 85\% no restante das bases de dados teve-se na Scopus 569 citações que corresponde aos artigos que foram citados pelo menos 10 vezes e na WoS obteve-se 305 citações, ou seja, a seleção foi composta pelos artigos que foram citados 9 vezes ou mais, tendo na Scopus 23 artigos e na Web of Science 15 artigos.

Para finalizar a seleção dos artigos, fez-se necessário à validação do tamanho da amostra que representará todos os dados que foram coletados; logo tem-se na composição final do portfólio 9 artigos da base Scopus, 5 da WoS e 3 da SciELO (Quadro 1).

Quadro 1. Artigos selecionados da base de dados Scopus, WoS e SciELO

\begin{tabular}{|c|c|c|c|c|}
\hline Títulos & Periódicos & Autores & $\begin{array}{c}\text { Ano } \\
\text { Publicação } \\
\end{array}$ & $\begin{array}{c}\mathbf{N}^{\mathbf{o}} \\
\text { citações }\end{array}$ \\
\hline \multicolumn{5}{|c|}{ SCOPUS } \\
\hline $\begin{array}{c}\text { Highly efficient and selective } \\
\text { wastewater by magnetically } \\
\text { recoverable } \mathrm{La}(\mathrm{OH}) 3 / \mathrm{Fe} 3 \mathrm{O} 4 \\
\text { nanocomposites phosphate removal } \\
\text { from }\end{array}$ & Water Research & $\begin{array}{l}\text { Wu B., Fang L., Fortner } \\
\text { J.D., Guan X., Lo I.M.C. }\end{array}$ & 2017 & 75 \\
\hline $\begin{array}{l}\text { Phosphorus recovery and reuse from } \\
\text { waste streams }\end{array}$ & $\begin{array}{l}\text { Advances in } \\
\text { Agronomy }\end{array}$ & $\begin{array}{l}\text { Karunanithi R., Szogi } \\
\text { A.A., Bolan N., Naidu } \\
\text { R., Loganathan P., Hunt } \\
\text { P.G., Vanotti M.B., } \\
\text { Saint C.P., Ok Y.S., } \\
\text { Krishnamoorthy S. }\end{array}$ & 2015 & 46 \\
\hline $\begin{array}{c}\text { Treatment of African catfish, Clarias } \\
\text { gariepinus wastewater utilizing } \\
\text { phytoremediation of microalgae, } \\
\text { Chlorella sp. with Aspergillus niger } \\
\text { bio-harvesting }\end{array}$ & $\begin{array}{l}\text { Bioresource } \\
\text { Technology }\end{array}$ & $\begin{array}{l}\text { Nasir N.M., Bakar } \\
\text { N.S.A., Lananan F., } \\
\text { Abdul Hamid S.H., Lam } \\
\text { S.S., Jusoh A. }\end{array}$ & 2015 & 35 \\
\hline $\begin{array}{c}\text { Comparative environmental impacts } \\
\text { of source-separation systems for } \\
\text { domestic wastewater management in } \\
\text { rural China }\end{array}$ & $\begin{array}{l}\text { Journal of Cleaner } \\
\text { Production }\end{array}$ & $\begin{array}{l}\text { Lam L., Kurisu K., } \\
\text { Hanaki K. }\end{array}$ & 2015 & 33 \\
\hline $\begin{array}{l}\mathrm{NaLa}(\mathrm{CO} 3) 2 \text { hybridized with } \mathrm{Fe} 3 \mathrm{O} 4 \\
\text { for efficient phosphate removal: } \\
\text { Synthesis and adsorption mechanistic } \\
\text { study }\end{array}$ & Water Research & Hao H., Wang Y., Shi B. & 2019 & 30 \\
\hline $\begin{array}{l}\text { Hydrated lanthanum oxide-modified } \\
\text { diatomite as highly efficient } \\
\text { adsorbent for low-concentration } \\
\text { phosphate removal from secondary } \\
\text { effluents }\end{array}$ & $\begin{array}{l}\text { Journal of } \\
\text { Environmental } \\
\text { Management }\end{array}$ & $\begin{array}{l}\text { Wu Y., Li X., Yang Q., } \\
\text { Wang D., Xu Q., Yao F., } \\
\text { Chen F., Tao Z., Huang } \\
\text { X. }\end{array}$ & 2019 & 30 \\
\hline
\end{tabular}

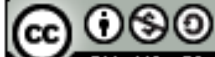


Citação (APA): Moreto, E. R., Porto, P. S. da S., \& Freitas, R. R. de. (2020). Análise bibliométrica de alternativas para remoção de nutrientes de águas residuais nas bases Scopus, Web of Science e Scielo. Brazilian Journal of Production Engineering, 6(7), $133-149$.

Journal of

Environmental

Science and

Health - Part A

novel zeolite/la

hybrid material prepared from coal fly ash

Removal of phosphate from surface and wastewater via electrocoagulation
Toxic/Hazardous

Substances and

Environmental

Engineering

Ecological
Engineering
Xie J., Lai L., Lin L., Wu D., Zhang Z., Kong

$\mathrm{H}$.

Franco D., Lee J.,

Arbelaez S., Cohen N., Kim J.-Y.

Bioresource Praveen P., Heng J.Y.P., Technology

2016

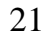

21 microalgae: Comparison of forward osmosis \& microfiltration

Tertiary wastewater treatment in

\begin{tabular}{|c|c|c|c|c|}
\hline \multicolumn{5}{|c|}{ Web of Science } \\
\hline $\begin{array}{l}\text { Phosphorus removal using a } \\
\text { microalgal biofilm in a new biofilm } \\
\text { photobioreactor for tertiary } \\
\text { wastewater treatment }\end{array}$ & Water Research & $\begin{array}{l}\text { Sukacova, Katerina; } \\
\text { Trtilek, Martin; Rataj, } \\
\text { Tomas }\end{array}$ & 2015 & 59 \\
\hline $\begin{array}{l}\text { Simultaneous nitrate and phosphate } \\
\text { removal from wastewater lacking } \\
\text { organic matter through microbial } \\
\text { oxidation of pyrrhotite coupled to } \\
\text { nitrate reduction }\end{array}$ & Water Research & $\begin{array}{l}\text { Li, Ruihua; Morrison, } \\
\text { Liam; Collins, Gavin; } \\
\text { Li, Aimin; Zhan, } \\
\text { Xinmin }\end{array}$ & 2016 & 43 \\
\hline $\begin{array}{c}\text { Adding value to the treatment of } \\
\text { municipal wastewater through the } \\
\text { intensive production of freshwater } \\
\text { macroalgae }\end{array}$ & $\begin{array}{l}\text { Algal Research- } \\
\text { Biomass Biofuels } \\
\text { and Bioproducts }\end{array}$ & $\begin{array}{c}\text { Cole, Andrew J.; } \\
\text { Neveux, Nicolas; } \\
\text { Whelan, Anna; Morton, } \\
\text { Jeff; Vis, Mark; de Nys, } \\
\text { Rocky; Paul, Nicholas } \\
\text { A. }\end{array}$ & 2016 & 22 \\
\hline $\begin{array}{c}\text { Nitrogen removal from urban } \\
\text { stormwater runoff by stepped } \\
\text { bioretention systems }\end{array}$ & $\begin{array}{l}\text { Ecological } \\
\text { Engineering }\end{array}$ & $\begin{array}{l}\text { Wang, Shumin; Lin, } \\
\text { Xiuyong; Yu, Hui; } \\
\text { Wang, Zhaodong; Xia, } \\
\text { Hongxia; An, Jinshuo; } \\
\text { Fan, Gongduan }\end{array}$ & 2017 & 17 \\
\hline $\begin{array}{c}\text { Phycoremediation strategies for rapid } \\
\text { tertiary nutrient removal in a waste } \\
\text { stream }\end{array}$ & $\begin{array}{l}\text { Algal Research- } \\
\text { Biomass Biofuels } \\
\text { and Bioproducts }\end{array}$ & $\begin{array}{c}\text { Filippino, Katherine C.; } \\
\text { Mulholland, Margaret } \\
\text { R.; Bott, Charles B. }\end{array}$ & 2015 & 16 \\
\hline \multicolumn{5}{|c|}{ SciELO } \\
\hline $\begin{array}{l}\text { Ambiance rose production and } \\
\text { nutrient supply in soil irrigated with } \\
\text { treated sewage }\end{array}$ & $\begin{array}{l}\text { Revista Brasileira } \\
\text { de Engenharia } \\
\text { Agrícola e } \\
\text { Ambiental }\end{array}$ & $\begin{array}{l}\text { Gabrielli, Giuliano, } \\
\text { Paixão, Jorge L. da, } \\
\text { Coraucci, Bruno, } \\
\text { Tonetti, Adriano L. }\end{array}$ & 2015 & 0 \\
\hline $\begin{array}{l}\text { Microalgae population dynamics in } \\
\text { photobioreactors with secondary } \\
\text { sewage effluent as culture medium }\end{array}$ & $\begin{array}{l}\text { Brazilian Journal } \\
\text { of Microbiology }\end{array}$ & $\begin{array}{l}\text { Marchello, Adriano E., } \\
\text { Lombardi, Ana T., } \\
\text { Dellamano-Oliveira, } \\
\text { Maria José, Souza, } \\
\text { Clovis W.O. }\end{array}$ & 2015 & 0 \\
\hline $\begin{array}{c}\text { Rice husks as a microbial source for } \\
\text { wastewater treatment }\end{array}$ & $\begin{array}{l}\text { Revista Brasileira } \\
\text { de Engenharia } \\
\text { Agrícola e } \\
\text { Ambiental } \\
\end{array}$ & $\begin{array}{l}\text { João, Jair J., Locks, } \\
\text { Luana, Vieira, José L., } \\
\text { Lucia, Eder A. }\end{array}$ & 2020 & 0 \\
\hline
\end{tabular}

Fonte: Autores, 2020.

Com os artigos selecionados, foi realizada uma análise dos principais resultados e conclusões dos autores dos portfólios sobre o tema em cada base de dados.

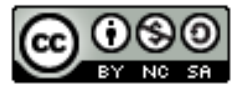


Citação (APA): Moreto, E. R., Porto, P. S. da S., \& Freitas, R. R. de. (2020). Análise bibliométrica de alternativas para remoção de nutrientes de águas residuais nas bases Scopus, Web of Science e Scielo. Brazilian Journal of Production Engineering, 6(7), 133-149.

\section{Scopus}

Hao, et al., (2019), Li, et al., (2019), Wu, et al., (2017) e Xi, et al., (2015) analisaram em laboratório a remoção de fosfato de águas residuais utilizando nano compósitos magneticamente regeneráveis.

Hao, et al., (2019) em seu estudo optaram pelo uso do lantânio modificado com zeólito, argila bentonita e sílica mesoporosa por quimissorção, ou seja, $\mathrm{NaLa}\left(\mathrm{CO}_{3}\right)_{2}$ hibridizado com $\mathrm{Fe}_{3} \mathrm{O}_{4}$; o método de remoção do fosfato utilizado foi o solvo térmico, cuja reação ocorre em meio não aquoso, possibilitando assim um maior controle do tamanho das partículas e a não formação de aglomerados indesejados no material; a partir disso obteve-se que a capacidade máxima de adsorção foi de 77,85 mg P por grama.

Seguindo com os estudos com lantânio, Li, et al., (2019) optaram pelo uso de compósitos de diatomita modificados com óxido de lantânio (La) hidratado; os mesmos foram fabricados por um método fácil e empregados como um adsorvente altamente eficiente para a remoção de fosfato de baixa concentração de simulação de efluentes secundários. O diatomita foi escolhido devido a sua estrutura macroporosa, (alta porosidade, 25-62\%) e sua capacidade de imobilizar o lantânio; segundo os autores Li, et al., (2019) um material de suporte poroso carregado La é capaz de remover o fosfato em baixa concentração inicial adequadamente. Como conclusão tem-se que $96 \%$ de $\mathrm{P}$ foi removido em um fosfato de baixa concentração (2 mg P / L) e obteve-se uma capacidade máxima de adsorção de P de 58,7mg P / L.

Wu, et al., (2017) fez uso do hidróxido de lantânio $\left(\mathrm{La}(\mathrm{OH})_{3}\right)$, que viabiliza a remoção do fosfato $\left(\mathrm{Fe}_{3} \mathrm{O}_{4}\right)$, juntamente com a magnetita que estimula a separação magnética das partículas sintetizando-os através dos métodos precipitação e hidrotérmico; o método de precipitação foi utilizado devido a sua simplicidade, alta eficiência e baixo custo e o método hidrotérmico por promover a cristalinidade dos produtos; como resultados os autores obtiveram uma eficiência na separação de $98 \%$ com uma capacidade de adsorção de 83,5 mg de fósforo (P) por grama, além de não causar toxicidade nas espécies aquáticas que se encontravam no ambiente de estudo.

Xie, et al., (2015) investigaram a eficiência de um adsorvente híbrido sintetizado composto por cinzas volantes de carvão, hidróxido de lantânio e zeólito (La-ZFA). Após a realização do experimento em laboratório concluiu-se que a capacidade máxima de remoção do material foi de 66,09 mg P / g, sendo que em águas de lagos o desempenho na remoção de $\mathrm{P}$ foi de 97,29\% e em uma estação de tratamento de efluentes alcançou-se 97,86\%.

Já Karunanithi, et al., (2015) optaram em realizar uma revisão bibliográfica com as principais tecnologias comerciais utilizadas para remoção de fosfato no mundo. Neste estudo chegou-se a 12 tecnologias das quais $58 \%$ delas são voltadas para a recuperação do fosfato como estruvita. Segundo os autores a estruvita é moderadamente solúvel em água e os íons de fosfato são dissolvidos rapidamente formando ligações com as partículas do solo que reduz a perda de P por escoamento superficial, a precipitação de cristais de estruvita apresenta custos bem menores, além de proporcionar a possibilidade de reutilização dos nutrientes.

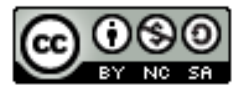


Citação (APA): Moreto, E. R., Porto, P. S. da S., \& Freitas, R. R. de. (2020). Análise bibliométrica de alternativas para remoção de nutrientes de águas residuais nas bases Scopus, Web of Science e Scielo. Brazilian Journal of Production Engineering, 6(7), 133-149.

Nasir, et al., (2015) testou a eficiência da microalga Chlorella sp. na remoção de nutrientes em águas residuais de aquicultura por meio da fito remediação de microalgas. A fito remediação é uma tecnologia que oferece um custo seguro e ambientalmente eficaz, pois são não intrusivos e limpos os vários tipos de contaminantes por meio das plantas verdes. Nasir et al. (2015) ao testarem várias doses de inoculação 0-60\% (v / v) para a fito remediação da Chlorella sp., chegaram ao resultado de que a dosagem ideal é de $30 \%$ (v / v) com um alcance de $98,7 \%$ na remoção da amônia e $92,2 \%$ na remoção de ortofosfatos.

Lam, Kurisu e Hanaki (2015) avaliaram o ciclo de vida e os impactos ambientais locais dos sistemas de separação na fonte de uso doméstico na China. Segundo eles, foram estudados quatro cenários; o primeiro foi o tratamento externo (B1), o segundo foi o tratamento interno (B2), o terceiro a separação na fonte (A1) e por último o uso do banheiro com descarga (A2); os impactos ambientais analisados foram o aquecimento global, acidificação e eutrofização. Os cenários que aumentam a recuperação de nutrientes (B2 e A2) mostraram benefícios significativos ao evitar o uso de fertilizantes minerais; no entanto, o sistema de separação de fontes (A1) apresentou o melhor desempenho em termos de impactos ambientais do ciclo de vida e melhor desempenho ambiental em termos de uso direto da água. Entretanto esse estudo possui limitações por ser realizado em laboratório e por ter deficiências na parte de dados estatísticos como relataram os autores.

Já Franco, et al., (2017) objetivaram em seu estudo um sistema de remoção de fosfato em escala de bancada que utiliza eletrocoagulação (CE). Eles examinaram os efeitos dos parâmetros de tratamento ( $\mathrm{pH}$ inicial, condutividade inicial, entrada de energia e concentração inicial de P) e a capacidade da CE de reduzir as concentrações de $\mathrm{P}$ nas águas superficiais e nas águas residuais tratadas. Após as análises, Franco et al. Obtiveram uma redução de 99\% em menos de 60 min da concentrações de $\mathrm{P}$ em soluções de fosfato, água de superfície, e efluentes de águas. Segundo eles a eficiência de remoção demonstrou ser diretamente proporcional à condutividade e potência fornecida.

Por último Praveen, Heng e Loh (2016) realizaram um estudo comparativo entre um foto biorreator de membrana baseado em microfiltração (FMM) e um foto biorreator de membrana osmótica baseado em osmose direta (FMO); eles foram operados com Chlorella vulgaris para tratamento contínuo de águas residuais terciárias. Biorreatores de membrana (MBR) funcionam através de uma combinação de remediação biológica e separação por membrana. Esses biorreatores oferecem as vantagens de reterem uma grande quantidade de biomassa, possuírem flexibilidade no processo e proporcionarem melhorias na qualidade do efluente. Ambos os biorreatores exibiram bom acúmulo de biomassa (acima de $2 \mathrm{~g} / \mathrm{L}$ ), embora o FMO tenha obtido melhor remoção de nutrientes devido às altas propriedades de rejeição das membranas. O FMO atingiu eficiências de remoção de nitrogênio e fósforo de $86-99 \%$ e $100 \%$, respectivamente, enquanto os valores correspondentes no FMM foram de 48-97\% e $46 \%$, respectivamente. Com base na entrada de energia, os custos operacionais totais para FMO foram 32-45\% mais altos do que para FMM, e o custo de filtração para FMO foi aproximadamente quatro vezes mais alto do que o FMM.

\section{@ $\odot \Theta \odot$}


Citação (APA): Moreto, E. R., Porto, P. S. da S., \& Freitas, R. R. de. (2020). Análise bibliométrica de alternativas para remoção de nutrientes de águas residuais nas bases Scopus, Web of Science e Scielo. Brazilian Journal of Production Engineering, 6(7), 133-149.

\section{Web of Science}

No portfólio de artigos do WoS, Sukacova, et al., (2015) objetivaram em seu estudo avaliar a capacidade de remoção de fósforo de um biofilme microalgal durante diferentes regimes de luz. Biofilmes foto tróficos são comunidades de multicamadas de foto autotróficos (cianobactérias e microalgas) e heterotróficos (bactérias, fungos e protozoários) que desempenham um papel fundamental na autopurificação de ecossistemas aquáticos. O foto biorreator foi operado por nove meses a cada ano durante um período de dois anos sem interrupção e sem necessidade de reinoculação. O biofilme de algas foi capaz de remover aproximadamente $97 \%$ do fósforo total das águas residuais durante 24 horas de iluminação artificial contínua.

Já Li, et al., (2016) optaram por investigar a eficiência de um biofiltro de desnitrificação autotrófico de pirrotita (BDAP) para a remoção simultânea de N e P de águas residuais sem matéria orgânica. Durante um período operacional de 247 dias, o BDAP removeu com eficiência o nitrato $\left(\mathrm{NO}_{3}{ }^{-}\right)$e o fosfato $\left(\mathrm{PO}^{3-}\right)$ simultaneamente de águas residuais que careciam de matéria orgânica, sendo que o NO3- foi removido por desnitrificação autotrófica com pirrotita como doador de elétrons e o fosfato foi removido principalmente através da formação de minerais secundários com $\mathrm{Fe} 3^{+}$.

Cole, et al., (2016) examinaram o uso de macroalgas de água doce como um processo de tratamento terciário em linha para estações de tratamento municipais de águas residuais de $29.000 \mathrm{~m}^{3}$. Uma monocultura de macroalga Edogonium intermedium foi inicialmente cultivada por um período de 3 meses em que a qualidade da água e a produtividade da biomassa foram quantificadas e a composição da biomassa caracterizada. Essas culturas foram então mantidas por mais 9 meses para determinar a produtividade média mensal da biomassa e a variação sazonal ao longo de um período de 12 meses. O cultivo de edogônio melhorou significativamente a qualidade da água descartada com uma redução de $36 \%$ no nitrogênio total e uma redução de $65 \%$ no fósforo total.

Wang, et al., (2017) fizeram uso do método de projeto de sistemas de biorretenção com foco em cidades montanhosas, pois ainda é raro na China e o nitrogênio é frequentemente liberado para o ambiente devido à falta de ambientes de desnitrificação. De 2015-2016, foram criadas 18 réplicas de sistemas de biorretenção escalonados, que incluíram uma camada de plantio de $400 \mathrm{~mm}$ de profundidade, uma camada de transição de $200 \mathrm{~mm}$ de profundidade e uma camada de cascalho de $200 \mathrm{~mm}$; foram 4 tipos diferentes de plantas sendo que cada uma se diferenciava pelo seu sistema radicular. Como resultados, os autores constataram que os dados de dois anos de operação mostraram que todos os sistemas forneceram tratamento satisfatório para o nitrogênio amoniacal $\left(\mathrm{NH}_{3}-\mathrm{N}\right)$.

Posteriormente Filippino Mulholland e Bott (2015) em seu estudo relataram uma nova estratégia de fito remediação que emprega algas para remover nitrogênio e fósforo de efluentes tratados antes da descarga. Segundo eles o uso de algas para remover nutrientes de águas residuais é uma estratégia atraente porque é barata, simples no design, sustentável e pode beneficiar o meio ambiente, pois as algas podem ser usadas como matéria-prima para a produção de biocombustíveis ou como ração animal ou fertilizante. Para tornar a fito 
Citação (APA): Moreto, E. R., Porto, P. S. da S., \& Freitas, R. R. de. (2020). Análise bibliométrica de alternativas para remoção de nutrientes de águas residuais nas bases Scopus, Web of Science e Scielo. Brazilian Journal of Production Engineering, 6(7), 133-149.

remediação em biorreatores viável dentro das instalações de recuperação de recursos hídricos municipais que operam em altas taxas de fluxo, fez-se uso de técnicas de imobilização, onde as algas foram incorporadas dentro de alginato de sódio; Como resultados obteve-se uma alta eficiência na remoção de nutrientes (até 100\%) em um tempo de retenção hidráulica de 6,5 horas.

\section{SciELO}

Gabrielli, et al., (2015) explicaram que efluentes de estações de tratamento de esgoto secundárias podem conter quantidade de nutrientes com potencial para causar eutrofização de corpos d'água. Devido isso, os autores avaliaram uma forma de diminuir esse influxo de nutrientes através do reaproveitamento agrícola por meio da irrigação de rosas da variedade Ambiance com o efluente de um reator anaeróbio e um filtro de areia intermitente (efluente nitrificado); Segundo Grabrielli, et al., (2015) o experimento durou 152 dias e obtiveram como resultados que uso de efluentes foi uma boa fonte de nitrogênio e uma fonte insuficiente de fósforo, o efluente anaeróbio era uma fonte razoável de potássio e o efluente nitrificado fornecia potássio em excesso.

Já Marchello, et al., (2015) monitoraram uma comunidade nativa de microalgas (Chlorella vulgaris) e bactérias coliformes em um efluente secundário de tratamento anaeróbio de esgoto municipal. Para analises, os autores aplicaram dois tratamentos (aerado e não aerado) para o cultivo de microalgas em condições semicontrolados em foto biorreatores semifechados em casa de vegetação. Como conclusão, as concentrações de nutrientes foram reduzidas apoiando o crescimento de microalgas em até $10^{7}$ células. $\mathrm{mL}^{-1}$ independente da aeração.

Por fim, João, et al., (2020) analisaram a casca de arroz para geração, crescimento e propagação de microrganismos, e posteriormente, utilizaram no tratamento de águas residuais para remoção de matéria orgânica e compostos de nitrogênio. Primeiramente, foram avaliados os seguintes tratamentos: água residual industrial (ARI), ARI + casca de arroz, ARI + serragem e ARI + folhas secas de árvores. Uma solução de $30 \mathrm{mg} \mathrm{L}^{-1}$ de $\mathrm{N}_{-} \mathrm{NO}_{3}$ contendo $3 \%$ $\left(\mathrm{v} \mathrm{v}^{-1}\right)$ de microrganismos obtidos da casca de arroz como substrato foi utilizada para avaliar a reutilização e desempenho da remoção de nitrato de nitrogênio. Segundo os autores, a demanda química de oxigênio, a demanda bioquímica de oxigênio, o nitrogênio total e os sólidos totais foram reduzidos acima de $80 \%$, além disso, o teste de reutilização demonstrou a estabilidade da atividade microbiana após sete dias de utilização.

\section{Comparação entre bases de dados}

Analisando as bases de dados comparativamente tem-se que o portfólio da base scopus contou com uma revisão bibliográfica sobre as principais tecnologias comerciais utilizadas para remoção de fosfato no mundo, 4 artigos utilizando o lantânio de diferentes formas, eletrocoagulação e dois artigos testando o uso da alga Chlorella em fito remediação e biorreatores.

Já a base WoS contou com o uso de algas em todos os artigos, sendo pesquisas de longa duração, pelo menos 8 meses e com resultados não tão especificados, ou seja, ambos os autores fizeram conclusões mais generalizadas. 
Citação (APA): Moreto, E. R., Porto, P. S. da S., \& Freitas, R. R. de. (2020). Análise bibliométrica de alternativas para remoção de nutrientes de águas residuais nas bases Scopus, Web of Science e Scielo. Brazilian Journal of Production Engineering, 6(7), 133-149.

$\mathrm{Na}$ base SciELO, a quantidade de artigos foi pequena comparativamente e contou com estudos sobre o reaproveitamento agrícola de água residual por meio da irrigação com o efluente de um reator anaeróbio e um filtro de areia intermitente, foto biorreatores com tratamento aerado e não aerado com a microalga chlorella e a utilização de casca de arroz para geração, crescimento e propagação de microrganismos, e posteriormente, utilizaram no tratamento de águas residuais para remoção de matéria orgânica e compostos de nitrogênio.

Pode-se destacar que os estudos em todas as bases foram realizados em laboratório e como sugestão de trabalhos futuros sugeriram a aplicação em situações reais. Pensando nisso, elaborou-se aqui uma Matriz SWOT (Quadro 2), para destacar os pontos positivos e negativos e com isso dar visar subsidiar uma continuidade nas pesquisa sobre o tema proposto.

Quadro 2. Matriz SWOT elaborada com base nas informações coletados no portfólio

\begin{tabular}{|c|c|}
\hline Positivo & Negativo \\
\hline Forças & Fraquezas \\
\hline $\begin{array}{l}\text { - Remoção de nutrientes das águas. } \\
\text { - Diminuição de contaminantes. } \\
\text { - Águas sem eutrofização. }\end{array}$ & $\begin{array}{l}\text { - Custos de implantação. } \\
\text { - Experimentos realizados apenas em laboratório. }\end{array}$ \\
\hline Oportunidades & Ameaças \\
\hline $\begin{array}{l}\text { - Reutilização dos nutrientes em outros } \\
\text { ambientes. } \\
\text { - Viabilização ambiental (estar de acordo com a } \\
\text { lei). } \\
\text { - Aproveitamento da água ou liberação dela para } \\
\text { o ambiente. }\end{array}$ & $\begin{array}{l}\text { - Carência de estudos publicados em âmbito } \\
\text { nacional. } \\
\text {-Experimentos em laboratórios não se adequem na } \\
\text { realidade devido as condições adversas serem } \\
\text { incontroláveis pelo ser humano. }\end{array}$ \\
\hline
\end{tabular}

Fonte: Autores, 2020.

\section{CONCLUSÃO}

Com base na análise bibliométrica realizada nas bases Scopus, Web of Science e SciELO foi possível visualizar o crescimento de pesquisas visando à solução de problemas ambientais causados pela liberação de nutrientes no meio ambiente. Isso, em torno de $40 \%$ em todas as bases analisadas, quando comparados os anos de 2015 a 2019.

Outra constatação é que grande parte dos artigos nacionais foram publicados em periódicos estrangeiros, mesmo assim o Brasil está em terceiro lugar em quantidade de publicações mundialmente. Entretanto esse fato pode vir a "enfraquecer" as revistas nacionais que abordam a temática.

Por fim, esse estudo destacou diferentes formas e tecnologias para remoção de nutrientes de águas resíduas considerando vários pontos, tais como, tecnologias mais baratas de implantação ou com alto custo, métodos de remoção de pequena ou grande escala, com adição de compósitos químicos para remoção ou uso de microalgas para absorção dos nutrientes, entre outros. Realizando assim um direcionamento das possíveis tecnologias de remoção de nutrientes ao redor do mundo foi impossível identificar qual delas pode ser mais eficiente, vantajosa ou menos onerosa, considerando sem dúvida outros pontos e novas análises possíveis com testes em campo.

\section{Agradecimentos}

Edital Fapes/Vale/Faperj No 01/2015 - Pelotização, Meio Ambiente E Logística.

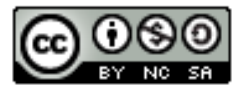


Citação (APA): Moreto, E. R., Porto, P. S. da S., \& Freitas, R. R. de. (2020). Análise bibliométrica de alternativas para remoção de nutrientes de águas residuais nas bases Scopus, Web of Science e Scielo. Brazilian Journal of Production Engineering, 6(7), 133-149.

\section{REFERÊNCIAS}

Barbetta, P. A. (2007). Estatística Aplicada Às Ciências Sociais. Florianópolis: Editora UFSC.

Boyle, F., \& Sherman, D. (2006). The product and its development. The Serials Librarian, Philadelphia, 49(3), 147-153.

Brasil. Ministério do Meio Ambiente. (2011). Conselho Nacional do Meio Ambiente Conama. Resolução No 430 de 13/05/2011 (Federal) - Dispõe sobre as condições e padrões de lança mento de efluentes complementam e alteram a Resolução $\mathrm{N}^{\circ}$ 357, de 17 de março de 2005, do Conselho Nacional do Meio Ambiente - CONAMA. 2011. Acesso em: 06 ago. 2020. Disponível em: 〈http://bit.ly/1FY24dj>.

Brasil. (2010). Política Nacional de Resíduos Sólidos. Lei No 12.305, de 2 de agosto de 2010. Presidência da República, Departamento da Casa Civil. Brasília. Acesso em: 20 abr. 2020. Disponível em: < http://www.planalto.gov.br/ccivil_03/leis/L9605.htm>.

Brasil. (1998). Lei $\mathrm{n}^{\circ}$ 9.605, de 12 de fevereiro de 1998. Dispõe sobre as sanções penais e administrativas derivadas de condutas e atividades lesivas ao meio ambiente, e dá outras providências. Diário Oficial da União, Brasília, 13 fev. 1998. Acesso em: 20 abr. 2020. Disponível em: < http://www.planalto.gov.br/ccivil_03/leis/L9605.htm>.

Cay, T., \& Uyan, M. (2013). Evaluation of reallocation criteria in land consolidation studies using the Analytic Hierarchy Process (AHP). Land Use Policy, Mersin, 30(1), 541- 548.

Cole, A. J., Neveux, N., Whelan, A., Morton, J., Vis, M., De Nys, R., \& Paul, N. A. (2016). Adding value to the treatment of municipal wastewater through the intensive production of freshwater macroalgae. Algal Research-Biomass Biofuels And Bioproducts. 20(1), 100-109.

Cui, W., Wu, Y., Liu, S., Wei, F., Zhou, M. X., \& Qu, H. (2010). Context preserving dynamic word cloud visualization. Pacific Visualization Symposium. Taipei, 1(1), 121-128, doi: 10.1109/PACIFICVIS.2010.5429600.

Dupont, W. D. ,\& Plummer, W. D. (1990). Power and sample size calculations: a review and computer program. Controlled clinical trials, Nashville, 11(2), 116-128.

Elsevier. (2020). Guia de referência rápida. Acesso em: 10 ago. 2020. Diponivel em: https://www.periodicos.capes.gov.br/images/documents/Scopus_Guia\%20de\%20refer\%C3\% AAncia\%20r\%C3\%A1pida_10.08.2016.pdf.

Filippino, K. C., \& Mulholland, M. R. (2015). Bott, C. B. Phycoremediation strategies for rapid tertiary nutrient removal in a waste stream. Algal Research-Biomass Biofuels And Bioproducts. 11(1), 125-133.

Franco D., Lee J., Arbelaez S., \& Cohen N. (2017). Kim J.Y. Removal of phosphate from surface and wastewater via electrocoagulation. Ecological Engineering. 108(1), 589-596. https://doi.org/10.1016/j.ecoleng.2017.07.031.

Gabrielli, G., Paixao, J. L. da, Tonetti, L., \& Coraucci, B. (2015). Ambiance rose production and nutrient supply in soil irrigated with treated sewage. Rev. bras. eng. agríc. ambient., Campina Grande , 19(8), 755-759. https://doi.org/10.1590/18071929/agriambi.v19n8p755-759.

Garcia, F. C. (2016). Identidade e imagem da marca: Uma análise comparativa em uma empresa do setor de serviços de telecomunicações. Dissertação (Mestrado em Administração. Universidade Federal de Uberlândia, Uberlândia. 
Citação (APA): Moreto, E. R., Porto, P. S. da S., \& Freitas, R. R. de. (2020). Análise bibliométrica de alternativas para remoção de nutrientes de águas residuais nas bases Scopus, Web of Science e Scielo. Brazilian Journal of Production Engineering, 6(7), 133-149.

Guedón, J. C. (2010). Acesso aberto e divisão entre ciência predominante e ciência periférica. Acessibilidade e visibilidade de revistas cientificas eletrônicas. São Paulo: SENAC São Paulo. Cengage Learning, 21-77.

Hao, H., Wang, Y., \& Shi, B. (2019). NaLa(CO3)2 hybridized with Fe3O4 for efficient phosphate removal: Synthesis and adsorption mechanistic study. Water Research. 155(1), 0111, 2019. https://doi.org/10.1016/j.watres.2019.01.049.

Joao, J. J., Locks, L., Vieira, J. L., \& Lucia, E. A. (2020). Rice husks as a microbial source for wastewater treatment. Rev. bras. eng. agríc. ambient., Campina Grande , 24(5), 343-347. https://doi.org/10.1590/1807-1929/agriambi.v24n5p343-347.

Karunanithi, R., Szogi, A.A., Bolan, N. N. R., Loganathan, P., Hunt, P.G., Vanotti, M. S., Ok, Y. S., \& Krishnamoorthy, S. (2015). Phosphorus Recovery and Reuse from Waste Streams. Advances in Agronomy. 131(1), 173-250. 10.1016/bs.agron.2014.12.005.

Lacerda, R. T. de O, Ensslin, L., \& Ensslin, S. R. (2012). Uma análise bibliométrica da literatura sobre estratégia e avaliação de desempenho. Gestão \& Produção, São Carlos, 19(1).

Lam A., Kurisu K., \& Hanaki, K. (2015). Comparative environmental impacts of sourceseparation systems for domestic wastewater management in rural China. Journal of Cleaner Production. 104(1), 185-198. https://doi.org/10.1016/j.jclepro.2015.04.126.

Li, R., Morrison, L., Collins, G., Li, A., \& Zhan, X. (2016). Simultaneous nitrate and phosphate removal from wastewater lacking organic matter through microbial oxidation of pyrrhotite coupled to nitrate reduction. Water Research. 96(1), 32-41.

Marchello, A. E., Lombardi, A. T., Dellamano-Oliveira, M. J., \& Souza, C. W.O. de. (2015). Microalgae population dynamics in photobioreactors with secondary sewage effluent as culture medium. Braz. J. Microbiol., São Paulo, 46(1), 75-84. Acesso em: 21 Set. 2020. Disponível em: <http://www.SciELO.br/SciELO.php?script= sci_arttext\&pid=S1517-83822015000100075\&lng=en\&nrm=iso>.

Mekonnen, M. M., \& Hoekstra, A. Y. (2018). Cargas globais de fósforo antropogênico para a água doce e pegadas hídricas cinza associadas e níveis de poluição da água: um estudo global de alta resolução. Recursos Hídricos Research, 54(1), 345-358. https://doi.org/10.1002/2017WR020448

Meneghini, R. (2003). O projeto SciELO (Scientific Electronic Library on Line) e a visibilidade da literatura científica "Periférica". Quím. Nova, São Paulo, 26(2), 155-156. https://doi.org/10.1590/S0100-40422003000200001.

Moreira, J. R., Vilan Filho, J. L., \& Mueller, S. P. M. (2015). Características e produção científica dos Grupos de pesquisa do CNPq/DGP nas áreas de Ciência da Informação e Museologia (1992 - 2012). Perspect. ciênc. inf., Belo Horizonte, 20(4), 93-106. Acesso em: 24 Ago. 2020. Disponível em: <http://www.SciELO.br/SciELO.php?script=sci_arttext\&pid=S141399362015000400093\&lng= en\&nrm=iso>.

Mugnaini, R. (2006). Caminhos para adequação da avaliação da produção científica brasileira: impacto nacional versus internacional. São Paulo, 253 p. Tese (Doutorado em Ciência da Informação) - Escola de Comunicações e Artes. Universidade de São Paulo. Disponível em: <http://poseca.incubadora.fapesp.br/portal/bdtd/2006/2006-domugnaini_rogerio.pdf $>$. Acesso em: 18 jul. 2020.

Nasir, N., Bakar, N., Lananan, F., Abdul, H. S. H., Lam, S. S., \& Jusoh, A. Treatment of African catfish, Clarias gariepinus wastewater utilizing phytoremediation of microalgae, 
Citação (APA): Moreto, E. R., Porto, P. S. da S., \& Freitas, R. R. de. (2020). Análise bibliométrica de alternativas para remoção de nutrientes de águas residuais nas bases Scopus, Web of Science e Scielo. Brazilian Journal of Production Engineering, 6(7), 133-149.

Chlorella sp. with Aspergillus niger bio-harvesting. Bioresource technology. 190(1). Doi: 10.1016/j.biortech.2015.03.023.

Natura. Relatório Annual. (2018). Disponivel em: https://static.rede.natura.net/html/2019/anatura/pdf/relatorio anual natura 2018.pdf. Acesso em: 06 out. 2020.

Pantano, G., Grosseli, G. M. Mozeto, A. A., \& Fadini, P. S. (2016). Sustentabilidade no uso do fósforo: uma questão de segurança hídrica e alimentar. Quím. Nova, São Paulo, 39(6), 732-740. Doi; http://dx.doi.org/10.5935/0100-4042.20160086.

Praveen P., Heng J.Y.P., \& Loh K. C. (2016). Tertiary wastewater treatment in membrane photobioreactor using microalgae: Comparison of forward osmosis \& microfiltration. $\begin{array}{llll}\text { Bioresource } & \text { Technology. 222(1), } & \text { 448-457. }\end{array}$ https://doi.org/10.1016/j.biortech.2016.09.124.

São Paulo. CETESB -Águas interiores. (2020). Acesso em: 17 set. 2020. Disponível em: https://cetesb.sp.gov.br/aguas-interiores/informacoes-basicas/tpos-de-agua/o-problema-daescasez-de-agua-no-mundo/.

São Paulo. CETESB -Poluição das águas subterrâneas. (2016). Acesso em: 17 set. 2020. Disponível em: https://cetesb.sp.gov.br/aguas-subterraneas/informacoes-basicas/poluicao-dasaguas-subterraneas/.

Souza, C. D. de. A organização do conhecimento: Estudo bibliométrico na base de dados ISI Web of Knowledge. Biblios: Journal of Librarianship and Information Science, [s 1.], n. 51, p.20-32, 4 jul. 2013.

Strehl, L. (2005). O fator de impacto do ISI e a avaliação da produção científica: aspectos conceituais e metodológicos. Ciência da informação. Brasília. 34(1), 19-27.

Sukacova, K,, Trtilek, M, \& Rataj, T. (2015). Phosphorus removal using a microalgal biofilm in a new biofilm photobioreactor for tertiary wastewater treatment. Water Research. 71(1), 55-63. Doi: https://doi.org/10.1016/j.watres.2014.12.049.

Trindade, P. B. C. B., \& Mendonca, A. S. F. (2014). Eutrofização em reservatórios - Estudo de caso: reservatório de Rio Bonito (ES). Eng. Sanit. Ambient., Rio de Janeiro , 19(3), 75282.Doi: http://dx.doi.org/10.1590/S1413-41522014019000000537.

Wang, S.; LIN, X.; Yu, H.; Wang, Z.; Xia, H.; An, J. \& Fan, G. (2017). Nitrogen removal from urban stormwater runoff by stepped bioretention systems. Ecological Engineering. 106(1), 340-348.

Wu, B., Fang, L., Fortner, J. D., Guan, X., \& Lo, I. M. C. (2017). Highly efficient and selective phosphate removal from wastewater by magnetically recoverable $\mathrm{La}(\mathrm{OH}) 3 / \mathrm{Fe} 3 \mathrm{O} 4$ nanocomposites, Water $\quad$ Research, 179-188. https://doi.org/10.1016/j.watres.2017.09.034.

Wu, Y., Li, X., Yang, Q., Wang, D., Xu, Q., Yao, F., Chen, F., Tao, Z., \& Huang, X. (2019). Hydrated lanthanum oxide-modified diatomite as highly efficient adsorbent for lowconcentration phosphate removal from secondary effluents. Journal of Environmental Management. 231(1), 370-379. https://doi.org/10.1016/j.jenvman.2018.10.059.

Xie, J., Lai, L., Lin, L., Wu, D., Zhang, Z., \& Kong, H. (2015). Phosphate removal from water by a novel zeolite/lanthanum hydroxide hybrid material prepared from coal fly ash. Journal of Environmental Science and Health. 50(12), 1298-1305. DOI: 10.1080/ 10934529.2015.1055159.

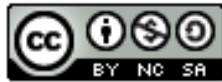

